\title{
Análisis psicométrico del Gratitude Questionnaire 6 (GQ-6) en población chilena*
}

Psychometric analysis of Gratitude Questionnaire 6 (GQ-6) in Chilean Population

Enviado: 12 de julio de 2014 | Revisado: 30 de abril de 2015 | Aceptado: 30 de abril de 2015

\author{
Marcos A. Carmona-Halty ** \\ MAURICIO MARÍN-GUTIERREZ*** \\ FRANCY BELMAR-SAAVEDRA $* * * *$ \\ Institución Universidad de Tarapacá, Chile
}

doi:10.11144/Javeriana.upsy14-3.apgq

Para citar este artículo: Carmona-Hally, M. A., Marín-Gutierrez, M \& Belmar-Saavedra, F. (2015). Análisis psicométrico del Gratitude Questionnaire 6 (GQ-6) en población chilena. Universitas Psychologica, 14(3), 865-872. http://dx.doi.org/10.11144/ Javeriana.upsy14-3.apgq

* Este reporte original de investigación contó con el apoyo de la Dirección General de Investigación de la Universidad de Tarapacá y del Convenio de Desempeño UTA-MINEDUC.

** Académico/Investigador de la Escuela de Psicología, Universidad de Tarapacá, Arica-Chile. Correo electrónico: mcarmonah@uta.cl

**** Estudiante Carrera de Psicología, Universidad de Tarapacá, Iquique-Chile. Correo electrónico: mauricio.a.marin@gmail.com

****** Estudiante Carrera de Psicología, Universidad de Tarapacá, Iquique-Chile. Correo electrónico: francybelmar.s@gmail.com (modificar mail)

\section{RESUMEN}

El objetivo de esta investigación es evaluar las propiedades psicométricas del Gratitude Questionnaire 6 (McCullough, Emmons \& Tsang, 2002) en población chilena. En el estudio participaron 307 hombres y 295 mujeres de diferente grupo etario. En primer lugar se estimó la confiabilidad y validez del constructo de la escala. En segundo lugar se analizaron indicadores de validez basados en la convergencia y divergencia con los instrumentos: Cuestionario de los cinco grandes, Escala de satisfacción vital, Escala de afectos positivos y negativos y el Test de orientación vital revisado. Los resultados indican adecuación del cuestionario para su utilización en la evaluación de la gratitud en población chilena.

Palabras clave

gratitud; fortalezas personales; propiedades psicométricas

\section{A B S T R A C T}

The objective of this research was to evaluate the psychometric properties of the Gratitude Questionnaire 6 (McCullough, Emmons \& Tsang, 2002) in Chilean population. In the study 307 men and 295 women of different age groups participated. We first estimated the reliability and construct validity of the scale. Secondly we analyzed the indicators convergent and divergent validity with these instruments: Big Five Questionnaire, Life Satisfaction Scale, Positive and Negative Affects Scale and Revised Life Orientation Test. The results indicate adequacy of the questionnaire for use in the evaluation of gratitude in Chilean population.

Keywords

gratitude; personal strengths; psychometric properties 


\section{Introducción}

La gratitud se ha definido como un rasgo afectivo que consiste en la disposición para reconocer y responder a los beneficios aportados por los demás en las propias experiencias positivas y logros personales (McCullough, Emmons \& Tsang, 2002). A pesar de que tradicionalmente la gratitud fue una de las emociones más ignoradas por las ciencias sociales (Solomon, 2004), trabajos recientes han encontrado evidencia que muestra los beneficios de experimentar esta emoción tanto para el propio individuo como para la sociedad (p. ej. Algoe, 2012; Algoe, Haidt \& Gable, 2008; Bartlett, Condon, Cruz, Baumann \& DeSteno, 2012; Bartlett \& DeSteno, 2006; Emmons \& Shelton, 2005; Fredrikson, 2004; Froh, Bono \& Emmons, 2010; Grant \& Gino, 2010; Lanham, Rye, Rimsky \& Weill, 2012; Seligman, Steen, Park \& Peterson, 2005; Toussaint \& Friedman, 2009).

En el contexto de la psicología positiva y el estudio de las fortalezas de carácter, se ha demostrado que es factible incluir la gratitud en el repertorio de las personas a través de una intervención deliberada (p. ej. Chan, 2010; 2011; Emmons \& McCullough, 2003; Martínez-Martí, Avia \& Hernández-Lloreda, 2010; Nelson, 2009; Ng \& Wong, 2013; Rash, Matsuba \& Prkachin, 2011). Lo anterior favorece su utilización en diversas intervenciones dirigidas a fomentar el bienestar y disminuir síntomas depresivos (p. ej. Cuadra-Peralta, Fuentes-Soto, MadueñoSoza, Veloso-Besio \& Bustos, 2012; Cuadra-Peralta, Veloso-Besio, Ibergaray \& Rocha, 2010; CuadraPeralta, Veloso-Besio, Puddu-Gallardo, SalgadoGarcía \& Peralta-Montecinos, 2012; Lambert \& Fincham, 2011; Lambert, Fincham \& Stillman, 2012; Lambert, Fincham, Stillman \& Dean, 2009; McCullough, Kilpatrick, Emmons \& Larson, 2001; McCullough, Kimeldorf \& Cohen, 2008; Park, Peterson \& Sun, 2013).

El Gratitude Questionnaire 6 (GQ-6) es un instrumento de auto reporte que mide la gratitud como rasgo disposicional en seis ítems. En cuatro estudios McCullough et al. (2002) concluyen que el cuestionario converge adecuadamente con informes de observación y una escala de medición de adjetivos de gratitud; posee excelentes propiedades psicométricas -incluyendo una estructura uni-factorial y alta consistencia interna- y se correlaciona de manera teóricamente esperada con medidas de afectos, conducta pro social y espiritualidad.

A pesar de que el GQ-6 se ha utilizado ampliamente para evaluar el constructo de gratitud (p. ej. Bernabé, García-Alandete \& Gallego-Pérez, 2013; Wood, Maltby, Stewart \& Linley, 2008), existe evidencia que señala un comportamiento estructural diferente al propuesto por sus autores, dependiente de la población en donde se utilice (Chen, Chen, Kee \& Tsai, 2008). Lo anterior sugiere, tal y como ocurre con otros constructos relativos al bienestar, que los instrumentos desarrollados para su medición se ven afectados por factores culturales provenientes de los países de origen (Alarcón, 2006; Castro, 2011). Adicionalmente, al día de hoy no se han realizado estudios sobre las características psicométricas del GQ-6 en población chilena. Por esta razón el objetivo del presente estudio es analizar las propiedades psicométricas del instrumento, siguiendo la línea de trabajos reciente que han contribuido a la validación de instrumentos relacionados al ámbito de la psicología positiva (p. ej. Dufey \& Fernández, 2012; Veliz, 2012; Vera-Villarroel, Urzúa, Pavez, Celis-Atenas \& Silva, 2012; Vera-Villarroel, CelisAtenas \& Córdova-Rubio, 2010; Vera-Villarroel, Córdova-Rubio \& Celis-Atenas, 2009).

\section{Método}

\section{Participantes y procedimiento}

En el presente estudio se entrevistó a un total de 602 participantes ( 307 hombres y 295 mujeres) con una edad media de 41.31 años $(D E=17.86)$. El procedimiento incluyó el análisis lingüístico del instrumento con la finalidad de detectar alguna dificultad en su comprensión; el contacto con los participantes en sus respectivos lugares de trabajo o estudios; la formalización de su participación a través de un consentimiento escrito que incluía los objetivos y aspectos generales del estudio; $y$ finalmente 
la aplicación del instrumento de manera individual siguiendo un procedimiento de lápiz y papel.

\section{Instrumentos}

Cuestionario de Gratitud (GQ-6) Se utilizó la versión en español del Gratitude Questionnaire 6, desarrollada por McCullough et al. (2002). El cuestionario se compone de seis ítems que evalúan la disposición al agradecimiento (p. ej. 'tengo mucho en la vida por lo que estar agradecido') en un formato de respuesta de tipo Likert con alternativas comprendidas entre uno (totalmente en desacuerdo) y siete (totalmente de acuerdo). La escala muestra una solución uni factorial y adecuados índices de confiabilidad en muestras de estudiantes universitarios, población general, niños y adolescentes (McCullough et al., 2002; McCullough, Tsang \& Emmons, 2004; Froh et al., 2011).

Inventario de los 5 grandes (BFI) Se utilizó la versión en español del Big Five Inventory compuesto por 44 ítems que evalúan las dimensiones del modelo de los 5 grandes: extroversión, afabilidad, responsabilidad, neuroticismo y apertura a la experiencia. Los ítems se responden en base a una escala tipo Lickert con puntuaciones comprendidas entre uno (muy en desacuerdo) y cinco (muy de acuerdo) en los cuales se solicita a la persona que responda en qué medida cada una de las afirmaciones le describen (p. ej. "es temperamental, de humor cambiante"). La escala cuenta con adecuadas propiedades psicométricas evaluadas en diversas muestras (Benet-Martínez \& John, 1998; Caprara, Barbaranelli, Borgogni \& Perugini, 1993; John \& Srivastava, 1999).

Escala de satisfacción vital (SWLS) Se utilizó la traducción al español de la Satisfaction With Life Scale (Diener, Emmons, Larsen \& Griffin, 1985), realizada por Atienza, Pons, Balaguer y GarcíaMerita (2000). La escala evalúa de manera global la satisfacción con la vida a través de cinco ítems (p. ej. "las condiciones de mi vida son excelentes") con puntuaciones comprendidas entre uno (totalmente en desacuerdo) y siete (totalmente de acuerdo). En población chilena se han encontrado adecuados indicadores de fiabilidad y una estructura uni factorial que explica un porcentaje significativo de la varianza del constructo (Vera-Villarroel et al., 2012).

Escalas de afecto positivo y negativo (PANAS) Se utilizó la traducción al español del Positive and Negative Affect Schedule (Watson, Clark \& Tellegen, 1988) desarrollada por Robles y Páez (2003), que incluye dos apartados: afecto como estado y afecto como rasgo. En el presente estudio se utilizó únicamente el apartado que define afecto como rasgo, el cual consta de 20 reactivos $(10$ de ellos evalúan el afecto positivo y los 10 restantes el afecto negativo) con opciones de respuesta entre uno (muy poco o nada) y cinco (extremadamente). En población chilena se han encontrado adecuados indicadores de fiabilidad y validez (Dufey \& Fernández, 2012).

Test de orientación vital revisado (LOT-R). Se utilizó la versión española del Life Orientation Test Revised Version (Scheier, Carver \& Bridges, 1994), desarrollada por Otero, Luengo, Romero, Gómez y Castro (1998). Se compone de seis ítems que miden el optimismo disposicional, tres de ellos redactados en sentido negativo (p. ej. "rara vez cuento con que me pasen cosas buenas") que deben ser invertidos para obtener la puntuación total. La escala posee un formato de respuesta entre cero (totalmente en desacuerdo) y cuatro (totalmente de acuerdo). En población chilena se obtuvo una consistencia interna aceptable (Vera-Villarroel et al., 2009).

\section{Análisis estadísticos}

En primer lugar se realizaron análisis descriptivos y comparación de medias a partir del sexo de los participantes. Luego se estimó la consistencia interna de la escala a través del coeficiente alfa de Cronbach y la correlación ítem-total. Posteriormente se dividió la muestra aleatoriamente en dos grupos de 301 participantes cada uno. Con el primer grupo se realizó un análisis factorial exploratorio y con el segundo, un análisis factorial confirmatorio. Finalmente se llevaron a cabo análisis de correlación para evaluar validez basada en la convergencia y divergencia utilizando los instrumentos descritos anteriormente. 


\section{Resultados}

\section{Estadísticos descriptivos}

En la Tabla 1 se muestran los estadísticos descriptivos y los resultados de la comparación de medias a partir de la variable sexo. En este último se observa la existencia de diferencias estadísticamente significativas que indican que las mujeres experimentan mayores niveles de gratitud que los hombres.

\section{Análisis de fiabilidad}

En la Tabla 2 se muestran los valores de la correlación item-total del GQ-6 y el índice alfa de Cronbach.

\section{Análisis factorial exploratorio (AFE)}

Dado que las variables contenidas en el GQ-6 no mostraron una distribución normal, se seleccionó el método de extracción de mínimos cuadrados generalizados y como criterio de extracción de factores, la regla de Kaiser-Guttman de auto-valor superior o igual a la unidad (Ruiz \& San Martín, 1992). La medida de adecuación muestral de Kaiser-MeyerOlkin $(\mathrm{KMO})$ indica una excelente adecuación (0.90), mientras que la Prueba de esfericidad de Bartlett indica que el modelo factorial es adecuado (986.95). El porcentaje de varianza explicada es de $65.88 \%$, las cargas factoriales pueden verse en la Tabla 3.

TABLA 1.

Medias y DT del GQ-6 en población general chilena

\begin{tabular}{ccccc}
\hline & Media (DT) & Hombres (DS) & Mujeres (DS) & $\mathrm{t}$ \\
\hline Ítem 1 & $5.70(1.27)$ & $5.55(1.32)$ & $5.86(1.20)$ & $-2.68^{* *}$ \\
Ítem 2 & $5.36(1.39)$ & $5.16(1.44)$ & $5.55(1.30)$ & $-3.04^{* *}$ \\
Ítem 3 & $5.14(1.23)$ & $5.11(1.29)$ & $5.16(1.16)$ & -0.46 \\
Ítem 4 & $5.23(1.38)$ & $5.05(1.50)$ & $5.40(1.25)$ & $-2.68^{* *}$ \\
Ítem 5 & $5.66(1.33)$ & $5.53(1.40)$ & $5.79(1.25)$ & $-2.10^{* *}$ \\
Ítem 6 & $5.06(1.16)$ & $4.95(1.18)$ & $5.17(1.13)$ & $-2.10^{* *}$ \\
Total & $5.36(1.09)$ & $5.22(1.16)$ & $5.49(1.00)$ & $-2.61^{* *}$ \\
\hline
\end{tabular}

$* * \mathrm{p}<0.001$

Fuente: elaboración propia

TABla 2.

Correlación item-total y Alpha de Cronbach del QG-6

\begin{tabular}{cc}
\hline & Correlación ítem-total \\
\hline Ítem 1 & 0.84 \\
Ítem 2 & 0.83 \\
Ítem 3 & 0.66 \\
Ítem 4 & 0.77 \\
Ítem 5 & 0.79 \\
Ítem 6 & 0.70 \\
Alpha de Cronbach & 0.75 \\
\hline
\end{tabular}

Fuente: elaboración propia
TABLA 3.

Cargas factoriales del GQ-6 método mínimos cuadrados generalizados

\begin{tabular}{cc}
\hline & Factor 1 \\
\hline Ítem 1 & 0.90 \\
Ítem 2 & 0.89 \\
Ítem 3 & 0.66 \\
Ítem 4 & 0.83 \\
Ítem 5 & 0.85 \\
Ítem 6 & 0.70 \\
\hline
\end{tabular}

Fuente: elaboración propia 


\section{Análisis factorial confirmatorio (AFC)}

Dados los resultados del AFE, el modelo original fue sometido a un AFC con el segundo grupo de la muestra. Los indicadores de ajuste del modelo unifactorial se muestran en la Tabla 4.

Validez basada en la

convergencia y divergencia

Para evaluar validez basada en la convergencia del modelo estudiado, se utilizó la escala de satisfacción vital (SWLS), el test de orientación vital revisado (LOT-R), la escala de afectos positivos del PANAS y las sub escalas de afabilidad y extraversión del inventario de los cinco grandes (BFI). El análisis de correlación de Pearson arrojó valores estadísticamente significativos para todas las variables, un efecto grande para satisfacción vital, $r(602)=$ $0.58, p=0.00$; optimismo $r(602)=0.57, p=0.00$; y afabilidad, $r(602)=0.52, p=0.00 ;$ y moderado para afectos positivos $r(602)=0.45, p=0.00 ; \mathrm{y}$ extroversión $r(602)=0.46, p=0.00$.
Para evaluar la validez divergente del GQ-6 se utilizó la escala de afectos negativos del PANAS, junto a la subescala de neuroticismo del BFI. Los resultados del análisis de correlación de Pearson indicaron relaciones inversas y moderadas con las medidas de afectos negativos, $r(602)=-0.37, p=$ 0.00 ; y neuroticismo, $r(602)=-0.30, p=0.00 \mathrm{La}$ matriz de correlación de todas las variables contenidas en el estudio se puede apreciar en la Tabla 5.

\section{Discusión}

El objetivo de esta investigación fue evaluar las propiedades psicométricas del GQ-6 en población general chilena. La evaluación de la consistencia interna fue adecuada ( $a l f a=0.75$ ), alcanzando valores similares a los obtenidos en estudios llevados a cabo en otros contextos (Bernabé et al., 2013; Chen et al., 2009; Froh et al., 2011). Además, la correlación ítem-total arrojó relaciones significativas según el estándar propuesto por Cohen (1992) en todos los ítems del cuestionario. En cuanto a la validez del constructo, del análisis factorial

TABLA 4.

Indicadores de bondad de ajuste del GQ-6

\begin{tabular}{ccccccccc}
\hline $\mathrm{X}^{2}$ & $\mathrm{gl}$ & $\mathrm{X}^{2} / \mathrm{gl}$ & $\mathrm{NFI}$ & $\mathrm{CFI}$ & TLI & IFI & RFI & RMSEA \\
\hline 69.90 & 9 & 7.78 & 0.97 & 0.97 & 0.93 & 0.97 & 0.92 & $0.12^{* *}$ \\
\hline
\end{tabular}

** $p<0.001$

Fuente: elaboración propia

TABla 5.

Matriz de correlación de las variables evaluadas

\begin{tabular}{lccccccc}
\hline & 1 & 2 & 3 & 4 & 5 & 6 & 7 \\
\hline 1. GQ-6 & & & & & & & \\
2. SWLS & $0.58^{* *}$ & & & & & & \\
3. LOT-R & $0.57^{* *}$ & $0.46^{* *}$ & & & & & \\
4. A. positivos & $0.46^{* *}$ & $0.40^{* *}$ & $0.43^{* *}$ & & & & \\
5. A. negativos & $-0.37^{* * *}$ & $-0.34^{* * *}$ & $-0.39^{* *}$ & -0.08 & & & \\
6. Extroversión & $0.46^{* *}$ & $0.45^{* *}$ & $0.45^{* *}$ & $0.47^{* *}$ & $-0.29^{* *}$ & & \\
7. Afabilidad & $0.8^{* *}$ & $0.51^{* *}$ & $0.44^{* *}$ & $0.43^{* *}$ & $-0.40^{* *}$ & $0.45^{* * *}$ & \\
8. Neuroticismo & $-0.0^{* *}$ & $-0.34^{* *}$ & $-0.41^{* * *}$ & $-0.36^{* *}$ & $0.52^{* *}$ & $-0.41^{* *}$ & $-0.41^{* *}$ \\
\hline
\end{tabular}

$* * \mathrm{p}<0.001$

Fuente: elaboración propia

| Universitas Psychologica | V. 14 | No.3 | Julio-septiembre | 2015 | 869 
exploratorio emerge un único factor que explica un porcentaje importante de varianza, el cual se replica en un análisis factorial confirmatorio con adecuados índices de bondad de ajuste, similares a los reportados por otros autores (p. ej. Bernabé et al., 2013; Chen, et al. 2009). La validez basada en la convergencia y divergencia del GQ-6 muestra relaciones en la línea de lo esperado teóricamente y acorde con lo reportado en trabajos previos (Chen et al., 2009; McCullough et al., 2002; Toussaint y Friedman, 2009). En base a lo anterior, la evidencia indica la adecuación del GQ-6, como una medida confiable y válida para el estudio de la gratitud en población chilena.

Direcciones futuras de investigación podrían ampliar los indicadores de validez de esta escala utilizando criterios de validez temporal, convergencia con medidas alternativas de gratitud y reportes externos que ayuden a complementar los indicadores aquí presentados.

\section{Referencias}

Alarcón, R. (2006). Desarrollo de una escala factorial para medir la felicidad. Revista Interamericana de Psicología, 40, 99-106.

Algoe, S. B. (2012). Find, remind and bind: The functions of Gratitude in every relationship. Social and Personality Psychology Compass, 6(6), 455-469.

Algoe, S. B., Haidt, J., \& Gable, S. L. (2008). Beyond reciprocity: Gratitude and relationships in everyday life. Emotion, 8(3), 425-429.

Atienza, F. L., Pons, D., Balaguer, I., \& García-Merita, M. L. (2000). Propiedades psicométricas de la Escala de Satisfacción con la Vida en adolescentes. Psicothema, 12(2), 314-319.

Bartlett, M. Y., Condon, P., Cruz, J., Baumann, J., \& DeSteno, D. (2012). Gratitude: Prompting behaviors that build relationships. Cognition and Emotion, 26, 2-13.

Bartlett, M. Y., \& DeSteno, D. (2006). Gratitude and prosocial behavior: Helping when it costs you. Psychological Science, 17, 319-325.

Benet-Martinez, V., \& John, O. (1998). Los cinco grandes across cultures and ethnic groups: Multitrait multimethod analyses of the big five in
Spanish and English. Journal of Personality and Social Psychology, 75(3), 729-750.

Bernabé, G., García-Alandete, J., \& Gallego-Pérez, J. P. (2013). Análisis comparativo de dos modelos del Gratitude Questionnaire - Six Items Form. Revista Latinoamericana de Psicología, 45(2), 279-288.

Caprara, G. V., Barbaranelli, C., Bergogni, L., \& Perugini, M. (1993). The big five questionnaire: A new questionnaire to asses the five factor model. Personality and Individual Differences, 15, 281-288.

Castro, A. (2011). Las rutas de acceso al bienestar, relaciones entre bienestar hedónico y eudaemónico. Un estudio en población argentina. Revista Iberoamericana de Diagnostico y Evaluación Psicológica, 31, 37-57.

Chan, D. W. (2010). Gratitude intervention and subjective well-being among chinese school teachers in Hong Kong. Educational Psychology, 30(2), 139-153.

Chan, D. W. (2011). Burnout and life satisfaction: Does gratitude intervention make a difference among chinese school teachers in Hong Kong? Educational Psychology, 31(7), 809-823.

Chen, L. H., Chen, M., Kee, Y. H., \& Tsai, Y. (2009). Validation of the gratitude questionnaire (GQ) in taiwanese undergraduate students. Journal of Happiness Studies, 10, 655-664.

Cohen, J. (1992). A power primer. Psychological Bulletin, 112, 155-159.

Cuadra-Peralta, A., Fuentes-Soto, L., Madueño-Soza, D., Veloso-Besio, C., \& Bustos, Y. (2012). Mejorando clima organizacional y de aula, satisfacción vital y laboral. Fractal: Revista de Psicología, 24, 3-25.

Cuadra-Peralta, A., Veloso-Besio, C., Ibergaray, M., \& Rocha, M. (2010). Resultados de la psicoterapia positiva en pacientes con depresión. Terapia Psicológica, 28, 127-134.

Cuadra-Peralta, A. Veloso-Besio, C., Puddu-Gallardo, G., Salgado-García, P., \& Peralta-Montecinos, J. (2012). Effects of a Positive Program in Depressive Symptoms and Life Satisfaction in the Elderly. Psicología: Reflexao e Critica, 25(4), 644-652.

Diener, E., Emmons, R., Larsen, J., \& Griffin, S. (1985). The Satisfaction With Life Scale. Personality Assessment, 49(1), 71-75.

Dufey, M., \& Fernandez, A. M. (2012). Validez y confiabilidad del positive affect and negative affect 
Schedule (PANAS) en estudiantes universitarios chilenos. Revista Iberoamericana de Diagnostico y Evaluación Psicológica, 34, 157-173.

Emmons, R. A., \& McCullough, M. E. (2003). Counting blessings versus burdens: An experimental investigation of gratitude and subjective well-being in daily life. Journal of Personality and Social Psychology, 84, 377-389.

Emmons, R. A., \& Shelton, C. M. (2005). Gratitude and the science of positive psychology. En C. R. Snyder \& S. J. López (Eds.). Handbook of positive psychology (pp. 459-471). New York: Oxford University Press.

Fredrikson, B. (2004). Gratitude, like other positive emotions, broadens and builds. En R. A. Emmons, \& M. E. McCullough (Eds.). The psychology of gratitude (pp. 145-166). New York: Oxford University Press.

Froh, J. J., Bono, G., \& Emmons, R. (2010). Being grateful is beyond good manners: Gratitude and motivation to contribute to society among early adolescents. Motiv Emot, 34, 144-157.

Froh, J., Fan, J., Emmons, R., Bono, G., Huebner, E. S., \& Watkins, P. (2011). Measuring gratitude in youth: Assessing the psychometric properties of adult gratitude scales in children and adolescents. Psychological Assessment, 23(2), 311-324.

Grant, A. M., \& Gino, F. (2010). A little thanks goes a long way: Explaining why gratitude expressions motivate prosocial behavior. Journal of Personality and Social Psychology, 98(6), 946-955.

John, O., \& Srivastava, S. (1999). The big five trait taxonomy: History, measurement and theorical perspectives. En L. Pervin \& O. John (Eds.). Handbook of Personality: Theorical and research pp: 102-138. New York: Gifford Press.

Lambert, N. M., Fincham, F. D., Stillman, T. F., \& Dean, L. R. (2009). More gratitude, less materialism: The mediating role of life satisfaction. The Journal of Positive Psychology, 4(1), 32-42.

Lambert, N. M., \& Fincham, F. D. (2011). Expressing gratitude to a partner leads to more relationship maintenance behavior. Emotion, 11, 52-60.

Lambert, N. M., Fincham, F. D., \& Stillman, T. F. (2012). Gratitude and depressive symptoms: The role of positive reframing and positive emotion. Cognition and Emotion, 26(4), 615-633.
Lanham, M. E., Rye, M. S., Rimsky, L. S., \& Weill, S. R. (2012). How gratitude relates to burnout and job satisfaction in mental health professionals. Journal Of Mental Health Counseling, 34(4), 341-354.

Martínez-Martí, M. L., Avia, M. D., \& HernándezLloreda, M. J. (2010). The effects of counting blessings on subjective well-being: A gratitude intervention in a spanish sample. The Spanish Journal of Psychology, 13(2), 886-896.

McCullough, M. E., Emmons, R. A., \& Tsang, J. (2002). The gratefull disposition: A conceptual and empirical topography. Journal of Personality and Social Psychology, 82, 112-127.

McCullough, M. E., Tsang, J., \& Emmons, R. A. (2004). Gratitude in intermediate affective terrain: Links of grateful moods to individuals differences and daily emotional experience. Journal of Personality and Social Psychology, 86, 377-389.

McCullough, M. E., Kilpatrick, S. D., Emmons, R. A., \& Larson, D. B. (2001). Is gratitude a moral affect? Psychological Bulletin, 127(2), 249-266.

McCullough, M. E., Kimeldorf, M. B., \& Cohen, A. D. (2008). An adaptation for altruism? The social causes, social effects, and social evolution of gratitude. Current Directions in Psychological Science, 17(4), 281-285.

Nelson, C. (2009). Appreciating gratitude: Can gratitude be used as a psychological intervention to improve individual well-being? Counselling Psychology Review, 24(3/4), 38-50.

Ng, M., \& Wong, W. (2013). The differential effects of Gratitude and sleep on psychological distress in patients with chronic pain. Journal of Health Psychology, 18(2), 263-271.

Otero, J. M., Luengo, A., Romero, E. Gómez, J. A., \& Castro, C. (1998). Psicología de personalidad. Manual de prácticas. Barcelona: Ariel Practicum.

Park, N., Peterson, C., \& Sun, J. (2013). La Psicología Positiva: Investigación y aplicaciones. Terapia Psicológica, 31(1), 11-19.

Rash, J. A., Matsuba, M. K., \& Prkachin, K. M. (2011). Gratitude and well-being: Who benefits the most from a gratitude intervention? Applied Psychology: Health and Well-Being, 3(3), 350-369.

Robles, R., \& Páez, F. (2003). Estudio sobre la traducción al español y las propiedades psicométricas de las es- 
calas de afecto positivo y negativo (PANAS). Salud Mental, 26(1), 69-75.

Ruiz, M. A., \& San Martín, R. (1992). Una simulación sobre el comportamiento de la regla $\mathrm{K} 1$ en la estimación del número de factores. Psicothema, 4(2), 543-550.

Seligman, M. E. P., Steen, T. A., Park, N., \& Peterson, C. (2005). Positive psychology progress: Empirical validation of interventions. American Psychologist, 60, 410-421.

Scheier, M. F., Carver, C. S., \& Bridges, M. W. (1994). Distinguishing optimism from neuroticism (and trait, self-mastery, and self-esteem): A re-evaluation of Life Orientation Test. Journal of Personality and Social Psychology, 67, 1063-1078.

Solomon, R. C. (2004). Foreword. En R. A. Emmons, \& M. E. McCullough (Eds.). Psychology of Gratitude (pp. 5-9). New York, NY: Oxford University Press.

Toussaint, L., \& Friedman, P. (2009). Forgiveness, gratitude, and well-being: The mediating role of affect and beliefs. Journal Of Happiness Studies, 10(6), 635-654.

Veliz, A. (2012). Propiedades psicométricas de la Escala de Bienestar Psicológico y estructura factorial en universitarios chilenos. Psicoperspectivas, 11(2), 143-163.

Vera-Villarroel, P., Celis-Atenas, K., \& Córdova-Rubio, N. (2010). Evaluación de la felicidad: análisis psicométrico de la escala de felicidad subjetiva en población chilena. Terapia Psicológica, 29, 127-133.

Vera-Villarroel, P., Córdova-Rubio, N., \& Celis-Atenas, K. (2009). Evaluación del optimismo: un análisis preliminar del life orientation test versión revisada (LOT-R) en población chilena. Universitas Psychologica, 8, 61-67.

Vera-Villarroel, P., Urzúa, A., Pavez, P., Celis-Atenas, K., \& Silva, J. (2012). Evaluation of subjective wellbeing: Analysis of the satisfaction with life scale in chilean population. Universitas Psychologica, 11(3), 719-727.

Watson, D., Clark, L. A., \& Tellegen, A. (1988). Development and validation of brief measures of positive and negative affect: The PANAS scales. Journal of Personality and Social Psychology, 54(6), 1063-1070.

Wood, A. M., Maltby, J., Stewart, N., \& Linley, P. A. (2008). A social-cognitive model of trait and state levels of gratitude. Emotion, 8(2), 281-290. 